\title{
Remote sensing mapping of macroalgal farms by modifying thresholds in the classification tree
}

\section{Yuhan Zheng, Carlos M. Duarte, Jiang Chen, Dan Li, Zhaohan Lou \& Jiaping} Wu

To cite this article: Yuhan Zheng, Carlos M. Duarte, Jiang Chen, Dan Li, Zhaohan Lou \& Jiaping Wu (2018): Remote sensing mapping of macroalgal farms by modifying thresholds in the classification tree, Geocarto International, DOI: 10.1080/10106049.2018.1474272

To link to this article: https://doi.org/10.1080/10106049.2018.1474272

Accepted author version posted online: 07

May 2018.

Submit your article to this journal $[\pi$

Џ Article views: 3

Q View related articles $\sqsubset$

View Crossmark data 
Publisher: Taylor \& Francis

Journal: Geocarto International

DOI: http://doi.org/10.1080/10106049.2018.1474272

\section{Remote sensing mapping of macroalgal farms by modifying thresholds in}

\section{the classification tree}

Yuhan Zhenga,b, Carlos M. Duarte, , Jiang Chen ${ }^{\mathrm{a}}$, Dan Lie, Zhaohan Lou ${ }^{\mathrm{a}, \mathrm{b}}$, and Jiaping $\mathrm{Wu}^{\mathrm{a}, \mathrm{b}^{*}}$

${ }^{a}$ Ocean College, Zhejiang University, Zhoushan, China; ${ }^{b}$ Key Laboratory of Ocean

Observation-Imaging Testbed of Zhejiang Province, Zhejiang University, Zhoushan, China; 'King Abdullah University of Science and Technology (KAUST), Red Sea Research Center (RSRC), Thuwal, 23955-6900, Saudi Arabia; dThe University of Western Australia, Oceans Institute, 35 Stírling Hwy, Crawley, WA 6009, Australia; ${ }^{\mathrm{e} C o l l e g e}$ of Environment and Natural Resources, Zhejiang University, Hangzhou, China

*Correspondence: jw67@zju.edu.cn; Tel.: +86 5802092369

Yuhan Zheng: yuhanzheng@zju.edu.cn; Jiang Chen: chenjiangs@zju.edu.cn;

Carlos M. Duarte: carlos.duarte@kaust.edu.sa;

Dan Li: li65152@gmail.com; Zhaohan Lou:ywlnfn@163.com. 
Acknowledgement This research was partially funded by the State Oceanic Administration (Grant \# 529105-T21702, Strategy and Implementation of Blue Carbon Program in China, and Grant \# 529105-T01603, Sino-Australian joint research on measures and strategies of ocean eutrophication control).

\begin{abstract}
Remote sensing is the main approach used to classify and map aquatic vegetation, and classification tree (CT) analysis is superior to various classification methods. Based on previous studies, modified CT can be developed from traditional CT by adjusting the thresholds based on the statistical relationship between spectral features to classify different images without ground-truth data. However, no studies have yet employed this method to resolve marine vegetation. In this study, three Gao-Fen 1 satellite images obtained with the same sensor on January 30, 2014, November 5, 2014, and January 21, 2015 were selected, and two features were then employed to extract macroalgae from aquaculture farms from the seawater background. Besides, object-based classification and other image analysis methods were adopted to improve the classification accuracy in this study. Results show that the overall accuracies of traditional CTs for three images are $92.0 \%, 94.2 \%$ and $93.9 \%$, respectively, whereas the overall accuracies of the two corresponding modified CTs for images obtained on January 21, 2015 and November 5, 2014 are 93.1\% and 89.5\%, respectively. This indicates modified CTs can help map macroalgae with multi-date imagery and monitor the spatiotemporal distribution of macroalgae in coastal environments.
\end{abstract}

Keywords: macroalgae, modified classification tree, GF-1, classification accuracy

\title{
1. Introduction
}


Macroalgae dominate coastal waters around the world, particularly in rocky shores, and play an important role in maintaining coastal ecosystem balance (Troell et al. 1999; Sondak and Chung 2015) due to its high potential in reducing eutrophication and sequestrating carbon (Duarte et al. 2017; Xiao et al. 2017). It also provides as healthy food supply and medicinal ingredients in our daily life (Wei et al. 2013; Philippsen et al. 2014). For example, Kelp has high content in Iodine, essential to human health (Albericci, 1945) and brown algae is an important source of anti-cancer substances (Kim et al. 2010). The rapid growth and healthy nutrients of macroalgae favor high productivity and provide a significant commercial value (Abreu et al. 2011; Aitken et al. 2014). Macroalgae aquaculture makes up 27\% of total marine aquaculture production to date (Duarte et al. 2017). Among various countries, China is the largest producer and consumer of macroalgae, accounting for about two-thirds of the global production (Mazarrasa et al. 2014). There are seven macroalgae species now cultivated in China, namely Kelp, Porphyra, Undaria, Gracilaria, Eucheuma, Sargassum usiforme and Ulva. The total aquaculture macroalgal production increased by 8.6 -fold from $242,495 \mathrm{t}$ in 1983 to $2,089,153 \mathrm{t}$ in 2015 , and the corresponding farming area increased by 7.4-fold from 17,640 ha in 1983 to 130,564 ha in 2015 (China Fishery Statistical Yearbook, 1983-2015). Because macroalgal aquaculture has an outstanding relevance environmentally, ecologically and economically, there is an urgent demand for developing an efficient monitoring way to monitor farming areas, estimate its yield, obtain aquaculture information, and improve its governance. Currently used methods include field survey and manual reporting, which are time consuming and inefficient. Remote sensing (RS) technology is an ideal alternative because of its convenience, economy, and accuracy (Nellis et al. 1996; Vieira et al. 2012).

RS imagery is suitable for the identification of aquatic macrophytes (Silva et al. 2008; Wang et al. 2015). For example, Casal et al. (2011) mapped kelp forests on the south European Atlantic shelf by using SPOT-4. Roelfsema et al. (2014) identified the seagrass cover, species, and biomass with high-spatial-resolution satellite image data. Han et al. (2015) examined the changes in wetland in Poyang Lake, China in winter with Landsat imagery. Among classification methods utilized to distinguish aquatic vegetation, classification tree (CT) analysis is relatively widely used because of its high accuracy and capacity to incorporate artificial intelligence (Baker et al. 2006; Davranche et al. 2010; Khanna et al. 2011). In other words, CT is a white-box which provides insight into the classification process in the form of intelligible rules that users can employ. While other classifiers may also perform well in identifying macroalgae, such as Random Forest and Support Yector Machines, these are black-box approaches that do not yield insights into their classification procedure. However, most studies have established one CT for a specific time or constructed several CTs individually. The CT developed for one time period may also be unfeasible when this method is applied to other data due to the spectral or texture differences between the two periods. As such, employing single CT to map multi-date images is difficult without any adjustments of the CT structure or thresholds. Meanwhile, we need to collect the ground-truth data for each class to train and establish the CTs for different images. Several studies have attempted to modify CT thresholds when ground-based data are absent. Jiang et al. (2012) applied CT to classify and map aquatic vegetation in Taihu Lake, China with different satellite images. Zhao et al. (2012) also identified the aquatic vegetation 
distribution in Taihu Lake by utilizing modified CT results and suggested that modified CT is more stable than traditional CT to map aquatic vegetation in time periods. Furthermore, Luo et al. (2014) developed a new method to modify thresholds in CT and evaluated the classification accuracies of both traditional and modified CT. They found that modified models with linear adjustment performed well in mapping aquatic vegetation without ground-truth data. Studies have focused on lake ecosystems, but studies on coastal waters, especially coastal areas including macroalgal farms, have yet to be performed. As modifying thresholds in CT is of great advantage to map long-time series images, it is necessary to assess and apply this method in mapping macroalgal farms and monitoring their spatiotemporal distribution.

Coastal marine environments are complex due to the interaction between watershed inputs and ocean waters and the dynamics added by waves and wind. The rapidly expanding macroalgal farming areas and their important roles in marine environment and ecosystems (Duarte et al. 2017; Xiao et al. 2017) require efficient monitoring measures. Mapping macroalgae in coastal areas is still an issue, and no attempts at doing so using remote sensing analyzed by modified CTs have been reported. Thus, the aims of this study were to evaluate the applicability and to improve the performance of modified CTs in habitat mapping including macroalgal farms using high-spatial-resolutionimages.

\section{Materials and methods}

\subsection{Study area}

The study area is located in Dayu Bay, southeast of Zhejiang province, China (Figure 1a), with a macroalgae aquaculture area of about $30 \mathrm{~km}^{2}$. A ground view of the study area is shown in Figure 1(b), and the details about Porphyra farms can be seen in Figure 1c. Dayu Bay is a typical semi-closed bay with seyeral small islands near the bay mouth. The seawater in the bay is relatively calm, which is beneficial to macroalgae. Dayu Bay contains only Porphyra farms because of the high economic return of this macroalgae. Porphyra production in this area is contracted to more than 1,000 individuals and cultivated in rows on floating rafts. Porphyra cultivation in Dayu Bay usually starts in October of each year. After one to two months, the Porphyra can be reaped for the first harvest, and the entire growth period can last till March of the next year, and about 12 harvests per growth cycle are accomplished. In one cycle, Porphyra grows on the seawater surface and become exposed to air except during high tide. Considering this feature, we can choose the RS images with low tide and distinguish Porphyra farms from seawaters.

\subsection{Imagery data and pre-processing}

GF-1 is China's high-spatial-resolution RS satellite with four wide-field-of-view (WFV) cameras (Feng et al. 2016). Three GF-1 images over Dayu Bay obtained on January 30, 2014, November 5, 2014, and January 21, 2015 were adopted in our study, and the dataset details are listed in Table 1. All preprocessing procedures were conducted in ENVI 5.1 software, including image clipping, ortho-rectification, radiometric calibration, and atmospheric correction. Radiometric calibration is based on a former study that utilizes Landsat 8 Operational Land Imager images to cross calibrate the GF-1 WFV cameras and 
solve the problem caused by a large view angle (Feng et al. 2016). We implemented the Fast Line-of-Sight Atmospheric Analysis of Spectral Hypercubes (FLAASH) algorithm to remove the scattering components in the atmosphere and reflectance on the sea surface from the top of the atmosphere. Although the FLAASH atmospheric correction is commonly applied in land areas, studies show that it can also work appropriately in lake and marine environments (Kutser et al. 2006; Mishra et al. 2007; Pu et al. 2014). By considering our interested classes, we then masked the land areas for all images with the Normalized Difference Water Index (NDWI) (Gao 1996). In particular, the spectrum mixes with the seawater because Porphyra is farmed on the sea surface and interfered by seawater. We adjusted the NDWI threshold so that the Porphyra farms are not masked as land areas and instead divided into sea areas. Several studies also show that the principal component (PC) transform can strengthen image information and improve the classification accuracy for aquatic vegetation (Lee et al. 1990; Jia and Richards 1999; Yao et al. 2014). We then obtained four PC bands as our features in the ENVI software. At the end, we obtained mean values of four bands and four PC bands of each image to train the CTs.

\subsection{Traditional CT}

$\mathrm{CT}$ analysis is based on the dichotomous partitioning of the data at certain thresholds of the values of the explanatory variables, which determine the branch that a particular sample will follow (Jiang et al. 2012). Firstly, we need to determine the classification units in this study. Previous studies show that object-based classification works better than pixel-based classification in high spatial resolution imagery (Dorren et al. 2003; Myint et al. 2011; Chen et al. 2012; Jebur et al. 2013). We have compared these two methods in Porphyra farms identification in Dayu Bay, showing that object-based classification received approximately $10 \%$ higher accuracy than pixel-based classification (Zheng et al. 2017). Hence, we established our CTs based on the segmented objects rather than pixels of all layers and PCs. The multi-resolution segmentation based on the Fractal Net Evolution Approach was implemented in the Definiens Deyeloper, and the segmentation parameter can be adjusted by the heterogeneity criterion of the objects. Based on a series of trials and visual evaluation, we finally adopted the most suitable composition in our case: segmentation scale (10), compactness (0.5), and shape index (0.1). After obtaining the classification objects in our study area, J48 algorithm was employed to establish the CTs. The J48 algorithm has been widely applied in CT modeling, which can be used to perform the data mining task and create the most suitable CT for each image (Goethals et al. 2001; Kaur and Chhabra 2014; Sharma 2015). The J48 algorithm is based on C4.5, which selects a feature and splits the data into two subsets according to the highest normalized information gain (Zarkami 2011). This procedure repeats on each subset until all objects in this subset belongs to one class. The J48 algorithm has also been proven to be applicable to object-based classification (Vieira et al. 2012; Cordeiro and Rossetti 2015), and the entire process can be conducted in the Waikato Environment for Knowledge Analysis (WEKA) software (Hall et al. 2009). In this study, we selected segmented objects as samples and potential features, and then input them into the WEKA software to train the CTs. Based on the J48 algorithm, WEKA conducted the machine learning process to choose the most suitable classification features and calculate corresponding thresholds to establish CTs. Considering the spectrum and 
texture differences between the Porphyra farms and seawater, we set the mean layer values and previously obtained PC values as our potential features to train CTs. Mean layer values represent the mean spectral values of objects in different layers (bands). For example, mean layer1 (ML1), mean layer2 (ML2), mean layer3 (ML3) and mean layer4 (ML4) in this study indicate the spectral information of Band1, Band2, Band3 and Band4. A total of 430

Porphyra objects and 300 seawater objects for each image were selected randomly as our samples. The samples we chose are evenly distributed across the whole study area and include different growth stages of Porphyra which contributes to reduce the noise in training samples. Then all samples and potential features for each image were trained in the software to determine the stable CT structure and features. Finally, ML2 and principal component3 (PC3) were adopted to build CT for Image I, while ML2, PC3 and ML1 were selected for Image II. Corresponding thresholds were also calculated according to the algorithm. Figure 2 shows the traditional CTs for the two images (I and II) produced by the J48 algorithm in WEKA.

\subsection{Modified CT}

Owing to the multi-date imagery, CT thresholds for one image may not be appropriate for the others. Thus, we aimed to develop new CTs for Image II by modifying the thresholds of traditional CT for Image I. First, we must determine the correlations between two images. The CTs are influenced by intrinsic and extrinsic factors, such as phenological development, atmospheric conditions, water constituents, sun and view angles, and so on. These influences may be different on the same object in two images. Thus, obtaining good linear relationships between two images based on the values of features from the same object is difficult. However, the ranked values of feature in one image can be linearly related to the ranked values for the same feature in another image (Luo et al. 2014). In addition, our study area only contains one type of macroalgae (Porphyra); thus, we directly ranked the feature values of the selected samples (430 Porphyra objects and 300 seawater objects) of images on January 30, 2014 and January 21,2015 and tried to obtain the linear relationships of each feature between two images. Scatterplots for each feature (ML2 and PC3) of two images were created (Figure 3), and both of them show high linear relationships with overall $R^{2}>0.9$. The relationships of feature values can be expressed by mathematic equations, which are depicted in Table 2. Finally, thresholds in CT of image I were recalculated according to the equations we derived above to obtain the corresponding thresholds for Image II. New thresholds for CT in Image II are also listed in Table 2. Modified CT has the same structure with traditional CT but new thresholds.

\subsection{Validation for CTs}

On the basis of very high-spatial-resolution images on Google Earth (http://earth.google.com) (Zhu and Woodcock 2014), we validated both traditional and modified CTs. As there were no images with the same date of Image I and Image II available, we chose the images from Google Earth which have the closest dates with Image I and Image II. The images obtained on 31 December 2013 and 31 December 2014 were employed to assess the performance of each CT on Image I and Image II respectively. Then we randomly selected 810 Porphyra farm samples and 300 seawater samples for both Image 
I and Image II. This process was accomplished in eCognition software with the two referenced images from Google Earth. Although the number of seawater samples is only less than half of the Porphyra samples, the total area of seawater samples is almost the same with that of the Porphyra. And the samples to validate different CTs for the same images are the same, thus, we believe the samples are balanced and can be used to represent and compare the accuracies of different classification outcomes.

\section{Results}

\subsection{Classification results and accuracy assessments}

Figure 4 and Table 3 show the classification results and accuracy assessments of traditional CTs and modified CT. Figures $4(a)$ and $4(b)$ represent the outputs of traditional CTs for Images I and II, whereas Figure 4(c) is the modified result of Image II. Table 3 is the confusion matrix for three CTs. Figures $4(b)$ and 4(c) show that the modified result has no significant misclassifications compared with traditional results on the whole, thereby indicating that the modified CT can also work well in distinguishing Porphyra farms. Table 3 verifies this result again. The producer and user accuracies for Porphyra farms are all higher than $90 \%$ for both traditional and modified CTs; however, for seawater, the producer accuracies are all less than $90 \%$, thereby indicating that some omission errors occur in seawater class. The overall accuracies for traditional and modified CTs of Image II are $94.2 \%$ and $93.1 \%$, and the kappa coefficients are 0.879 and 0.856 , respectively. Thus, we conclude that even the result generated from modified CT is slightly poorer than traditional $\mathrm{CT}$, the overall accuracy and kappa coefficient are relatively high, thereby demonstrating that the modified CT can be used in Porphyra farms identification.

\subsection{Further validation}

Two images we used above are in the same month but different years that represent the same growth stage of Porphyra. As such, we verified our modified method by using the images with different months in one growth cycle of Porphyra, namely Image II (January 21, 2015) and Image III (November 5, 2014) to see how it works for different phenology. We also compared the results from traditional CT and modified CT for Image III. The classification results are shown in Figure 5 and the overall accuracies of them are 93.9\% (traditional CT) and $89.5 \%$ (modified CT) respectively. The modified classification result is not as good as the traditional one, which is lower than $90 \%$. Figure 5 shows that some omission errors occur in the northwest direction. By contrast, in the other parts of Figures 5(a) and 5(b), the classification results are markedly similar.

\section{Discussion}

The classification results showed that both traditional and modified CTs were able to classify the macroalgal farms vs. seawater with high confidence, thereby indicating that J48 data mining algorithm is feasible for macroalgal identification and traditional CT has the stable structure to be modified and works well in coastal areas. According to the training samples, J48 algorithm in WEKA software selected most suitable features and thresholds to establish CTs automatically. CTs for different images may have different structures and thresholds, as 
J48 algorithm always establishes CTs with the highest classification accuracy based on the training samples. If there are any changes in the samples, it may produce different CTs. However, plenty of studies show that CTs which have the same construction but modified thresholds can also achieve satisfying classification results (Zhao et al. 2012; Luo et al. 2014). In our study, we aim to explore the applicability of modified CT and provide a convenient way to map macroalgae without ground-truth data. Thus, it is not necessary to develop the CT with the highest accuracy. Although the classification accuracies of modified CTs are a little bit lower than the corresponding traditional CTs, it is still of great significance to apply modified CTs to obtain long-time series macroalgae distributions.

The CTs for our study area are mainly composed by three features selected by J48 algorithm, namely ML2, ML1, and PC3. As we mentioned before, mean layer value represented the spectral information of each band. ML2 and ML1 give the spectral information of green band and blue band respectively. Macroalgae has the similar spectrum characteristics with terrestrial vegetation, which shows relatively high/reflectance in green band, while seawater owns high reflectance in blue band. Besides, the PC3 values of Porphyra farms are higher than those of seawater referring to the statistical data of samples in our study. These contrasting characteristics between macroalgae and seawater verified the value of the $\mathrm{J} 48$ data mining algorithm in establishing the CTs.

Nevertheless, several factors may continue to influence the classification accuracy. First, the normal size of rafts in that area is nearly $5.4 \mathrm{~m} \times 15 \mathrm{~m}$, whereas the pixel of GF-1 image is $16 \mathrm{~m} \times 16 \mathrm{~m}$, larger than the raft unit. Thus, mixed pixels may exist in the images, especially for the raft edges that influence the CTs modeling and classification accuracy. Second, the arrangements of floating rafts are varied and depend on each farmer. For example, the floating rafts near the shore are arranged relatively messily but densely, whereas the others far from the coast are in regular rows. Thus, because of the poor spatial texture relationships and weak spectral information, identifying the messy Porphyra farms is relatively difficult. Figures 4 and 5 also reveal that most of the classification errors occur near the shore. Finally, although the entire growth period of Porphyra spans from October to March (the next year), Porphyra grows and floats on the sea surface for only roughly four months (November to February). Furthermore, the seeding times can differ slightly among individual farms, thus, capturing all Porphyra exposed clearly in one image is difficult, especially at the beginning of exposure time (November) and the harvest time (February). In our study, we used the CT of Image I to modify Image II. Both of them were acquired in January, when most of the Porphyra is above or near the sea surface; thus, the modified classification result is quite good and only slightly lower than the result generated from the J48 algorithm. However, when we turned to modify CTs with different months, the classification result is not as good as that before. In the northwest part of Image III, maybe the Porphyra has not grown or was already harvested prior to the image acquisition; the Porphyra cannot be seen and their spectral reflectance is akin to seawater. Thus, if we use the CT obtained in December to modify it, we will be unable to reach the ideal result compared with traditional CT. Furthermore, some intrinsic and extrinsic factors affect the performance of modified CTs (Zhao et al. 2012). Different view angles for WFV, changes of the surrounding environment such as the tide, wind, and water constituents (Nelson et al. 2006), as well as the differences of remotely sensed reflectance caused by atmospheric 
conditions (Dave 1980) account for the non-linear relationships between features $\left(\mathrm{ML}_{2}\right.$ and $\mathrm{PC}_{3}$ ). Thus, we ranked the values of features for two images to minimize the inevitable intrinsic and extrinsic influences, and obtained good linear relationships results (Figure 3) with correlation coefficients of more than 0.9 .

Although a few flaws remain in modified CTs compared with traditional CTs, this approach is convenient and no longer needs ground-truth data. If we apply this method into a large space or time scale in coastal areas, its advantages can be magnified and the few flaws can be ignored. Additionally, the validation result suggests to select images in the same phenology of macroalgae or classify them by using traditional J48 algorithm with groundtruth data to gain high accuracy. However, if these two conditions cannot be satisfied, the modified CT can still work with satisfactory results.

In further studies, we aim to solve the problem caused by phenology and to apply this method into broad areas, that is, to acquire the spatiotemporal distribution of macroalgae along the China coast waters. In this case, we must classify images from different sensors or satellites for different macroalgal species. Thus, the applicability of this method to different image sources and varied macroalgal species should be evaluated.

\section{Conclusion}

The classification maps generated by different CTs all show high overall accuracies and kappa coefficients. J48 data mining algorithm performs well in establishing the traditional $\mathrm{CTs}$, and the ranked values of each feature displayed good linear relationships for modifying the new thresholds. These results prove that both traditional CTs and modified CTs can be applied to GF-1 images for mapping macroalgal farming areas. The modified CTs do not need any ground-truth data and can be used for the previous year's imagery. This further suggests that this method is preferred for the same phenology of macroalgae. If it is applied to a broad area or a time series, it can be time saving and highly accurate. In conclusion, modifying thresholds in CTs can be used to identify macroalgae farms with high classification accuracy for different years without ground-truth data. This approach provides a feasible and efficient mechanism to obtain macroalgal farming information in long-time series.

\section{Disclosure statement}

No potential conflict of interest was reported by the authors.

\section{References}

Abreu, Maria H, Rui Pereira, Charles Yarish, Alejandro H Buschmann, and Isabel SousaPinto. 2011. "IMTA with Gracilaria vermiculophylla: productivity and nutrient removal performance of the seaweed in a land-based pilot scale system." Aquaculture 312(1):77-87.

Aitken, Douglas, Cristian Bulboa, Alex Godoy-Faundez, Juan L Turrion-Gomez, and Blanca Antizar-Ladislao. 2014. "Life cycle assessment of macroalgae cultivation and processing for biofuel production." J. Clean. Prod. 75: 45-56.

Albericci V J. 1945. "Rapid colorimetric estimation of iodine in kelp." Analyst, 70(70):474. Baker, Corey, Rick Lawrence, Clifford Montagne, and Duncan Patten. 2006. "Mapping 
wetlands and riparian areas using Landsat ETM+ imagery and decision-tree-based models." Wetlands 26(2):465-74.

Casal, G., N. Sánchez-Carnero, J. Freire, and E. Sánchez-Rodríguez. 2011. "Remote sensing with SPOT-4 for mapping kelp forests in turbid waters on the south European Atlantic shelf." Estuar. Coast. Shelf Sci. 91(3):371-8.

Chen, Gang, Geoffrey J. Hay, Luis M. T. Carvalho, and Michael A. Wulder. 2012. "Objectbased change detection." Int. J. Remote Sens. 33(14):4434-57.

Chen, Z., Z. Zhao, P. Gong, and B. Zeng. 2006. "A new process for the segmentation of high resolution remote sensing imagery." Int. J. Remote Sens. 27(22):4991-5001.

China Fishery Statistical Yearbook, 1983-2015.

Cordeiro, Carlos Leandro De Oliveira, and Dilce De Fátima Rossetti. 2015. "Mapping vegetation in a late Quaternary landform of the Amazonian wetlands using object-based image analysis and decision tree classification." Int. J. Remote Sens. 36 (13):3397-422.

Dave, J. V. 1980. "Effect of atmospheric conditions on remote sensing of a surface nonhomogeneity." Photogramm. Eng. Remote Sens. 46(9):1173-80.

Davranche, Aurélie, Gaëtan Lefebvre, and Brigitte Poulin. 2010. "Wetland monitoring using classification trees and SPOT-5 seasonal time series." Remote Sens. Environ. 114(3):552-62.

Dorren, Luuk K. A., Bernhard Maier, and Arie C. Seijmonsbergen. 2003. "Improved Landsat-based forest mapping in steep mountainous terrain using object-based classification." For. Ecol. Manage. 183(s1-3):31-46.

Duarte C M, Wu J, Xiao X, et al. "Can Seaweed Farming Play a Role in Climate Change Mitigation and Adaptation?" Front Mar Sci, $2017,4$.

Feng, Lian, Juan Li, Weishu Gong, Xi Zhao, Xiaoling Chen, and Xiaoping Pang. 2016. "Radiometric cross-calibration of Gaofen-1 WFV cameras using Landsat-8 OLI images: A solution for large view angle associated problems." Remote Sens. Environ. 174:56-68.

Gao, Bo Cai. 1996. "NDWI-A normalized difference water index for remote sensing of vegetation liquid water from space." Remote Sens. Environ. 58(3):257-66.

Goethals, P. , K. Gasparyan, and N Pauw, De. 2001. "River restoration simulations by ecosystem models predicting aquatic macroinvertebrate communities based on J48 classification trees."Mededelingen 66(66):213-7.

Hall, Mark, Eibe Frank, Geoffrey Holmes, Bernhard Pfahringer, Peter Reutemann, and Ian H. Witten. 2009. "The WEKA data mining software: an update." Acm Sigkdd Explorations Newsletter 11 (1): 10-8.

Han, Xingxing, Xiaoling Chen, and Lian Feng. 2015. "Four decades of winter wetland changes in Poyang Lake based on Landsat observations between 1973 and 2013." Remote Sens. Environ. 156: 426-37.

Jebur, Mustafa Neamah, Helmi Zulhaidi Mohd Shafri, Biswajeet Pradhan, and Mahyat Shafapour Tehrany. 2013. "Per-pixel and object-oriented classification methods for mapping urban land cover extraction using SPOT 5 imagery." Geocarto Int. 29 (7):792806.

Jia, Xiuping, and John A. Richards. 1999. "Segmented principal components transformation for efficient hyperspectral remote-sensing image display and classification." IEEE Trans. Geosci. Remote Sens. 37(1):538-42. 
Jiang, Hao, Zhao, Dehua, Ying, An, and Shuqing. 2012. "A Method for Application of Classification Tree Models to Map Aquatic Vegetation Using Remotely Sensed Images from Different Sensors and Dates." Sensors. 12:12437-12454.

Kaur, Gaganjot, and Amit Chhabra. 2014. "Improved J48 Classification Algorithm for the Prediction of Diabetes." Int. J. Comput. Appl. 98(22):13-7.

Khanna, S., M. J. Santos, S. L. Ustin, and P. J. Haverkamp. 2011. "An integrated approach to a biophysiologically based classification of floating aquatic macrophytes." Int. J. Remote Sens. 32(4):1067-94.

Kim E J, Park S Y, Lee J Y, et al. 2010. "Fucoidan present in brown algae induces apoptosis of human colon cancer cells." BMC Gastroenterol., 10(1):96.

Kutser, Tiit, Ian Miller, and David L. B. Jupp. 2006. "Mapping coral reef benthic substrates using hyperspectral space-borne images and spectral libraries." Estuar. Coast. Shelf Sci. 70(3):449-60.

Lee, J. B., A. S. Woodyatt, and M. Berman. 1990. "Enhancement of high spectral resolution remote-sensing data by a noise-adjusted principal components transform." IEEE Trans. Geosci. Remote Sens. 28(3): 295-304.

Liu, Yong, Ling Bian, Yuhong Meng, Huanping Wang, Shifu Zhang, Yining Yang, Xiaomin Shao, and Bo Wang. 2012. "Discrepancy measures for selecting optimal combination of parameter values in object-based image analysis." ISPRS J. Photogramm. Remote Sens. 68(1):144-56.

Luo, Juhua, Ronghua Ma, Hongtao Duan, Weiping Hu, Jinge Zhu, Wenjiang Huang, and Chen Lin. 2014. "A New Method for Modifying Thresholds in the Classification of Tree Models for Mapping Aquatic Vegetation in Taihu Lake with Satellite Images." Remote Sens. 6(8):7442-62.

Mazarrasa, I, Y. S. Olsen, E Mayol, N Marbà, and C. M. Duarte. 2014. "Global unbalance in seaweed production, research effort and biotechnology markets." Biotechnol. Adv. 32(5):1028-36.

Mishra, Deepak R., Sunil Narumalani, Donald Rundquist, Merlin Lawson, and R. Perk. 2007. "Enhancing the detection and classification of coral reef and associated benthic habitats: A hyperspectral remote sensing approach." J. Geophys. Res-Atmos. 112(C8):127-31.

Myint, Soe W., Patricia Gober, Anthony Brazel, Susanne Grossman-Clarke, and Qihao Weng. 2011. "Per-pixel vs. object-based classification of urban land cover extraction using high spatial resolution imagery." Remote Sens. Environ. 115(5): 1145-61.

Nellis, M.Duane, Kevin Price, Stephen Egbert, and Jiaping Wu. 1996. "Natural resource capability of CRP lands as Grasslands in Southwest Kansas: A remote sensing and GIS perspective." Geocart Int. 11(3):23-8.

Nelson, Stacy A. C., Kendra Spence Cheruvelil, and Patricia A. Soranno. 2006. "Satellite remote sensing of freshwater macrophytes and the influence of water clarity." Aquat. Bot. 85(4):289-98.

Philippsen, Aaron, Peter Wild, and Andrew Rowe. 2014. "Energy input, carbon intensity and cost for ethanol produced from farmed seaweed." Renew. Sust. Energ. Rev. 38(38):60923.

$\mathrm{Pu}$, Ruiliang, Susan Bell, and Cynthia Meyer. 2014. "Mapping and assessing seagrass bed 
changes in Central Florida's west coast using multitemporal Landsat TM imagery." Estuar. Coast. Shelf Sci. 149:68-79.

Roelfsema, Chris M., Mitchell Lyons, Eva M. Kovacs, Paul Maxwell, Megan I. Saunders, Jimena Samper-Villarreal, and Stuart R. Phinn. 2014. "Multi-temporal mapping of seagrass cover, species and biomass: A semi-automated object based image analysis approach." Remote Sens. Environ. 150(7):172-87.

Sharma, Priyanka. 2015. "Comparative Analysis of Various Decision Tree Classification Algorithms using WEKA." 3 (2): 684-90.

Silva, Thiago S. F., Maycira P. F. Costa, John M. Melack, and Evlyn M. L. M. Novo. 2008. "Remote sensing of aquatic vegetation: theory and applications." Environ. Monit. Assess. 140(1-3):131-45.

Sondak, Calvyn F. A., and Ik Kyo Chung. 2015. "Potential blue carbon from coastal ecosystems in the Republic of Korea." Ocean Sci. J. 50(1):1-8.

Troell, Max, Patrik Rönnbäck, Christina Halling, Nils Kautsky, and A Buschmann. 1999. "Ecological engineering in aquaculture: use of seaweeds for removing nutrients from intensive mariculture." J. Appl. Phycol. 11(1):89-97.

Vieira, Matheus Alves, Antonio Roberto Formaggio, Camilo Daleles Rennó, Clement Atzberger, Daniel Alves Aguiar, and Marcio Pupin Mello. 2012. "Object based image analysis and data mining applied to a remotely sensed Landsat time-series to map sugarcane over large areas." Remote Sens. Environ. 123:553-62.

Wang, Anqi, Jiadai Chen, Changwei Jing, Guanqiong Ye, Jiaping Wu, Zhixing Huang, and Chaosheng Zhou. 2015. "Monitoring the invasion of Spartina alterniflora from 1993 to 2014 with Landsat TM and SPOT 6 satellite data in Yueqing bay, China." PLoS One. 10(8): 0135538.

Wei, Na, Josh Quarterman, and Yong-Su Jin. 2013. "Marine macroalgae: an untapped resource for producing fuels and chemicals." Trends Biotechnol. 31(2):70-7.

Xiao X, Agusti S, Lin F, et al. "Nutrient removal from Chinese coastal waters by large-scale seaweed aquaculture." Sci Rep, 2017, 7:46613.

Yao, Aidong, Xiaoyang Cao, and Yiming Feng. 2014. "Remote-sensing Model for Estimating the Size of Gobi Surface Gravel Based on Principal Components Analysis." Journal of Desert Research. 34(5):1215-1221.

Zarkami, R. 2011. "Application of classification trees-J48 to model the presence of roach (Rutilus rutilus) in rivers." Caspian J. Env. Sci. 9(2):189-98.

Zhao, Dehua, Hao Jiang, Tangwu Yang, Ying Cai, Delin Xu, and Shuqing An. 2012. "Remote sensing of aquatic vegetation distribution in Taihu Lake using an improved classification tree with modified thresholds." J. Environ. Manage. 95(1): 98-107.

Zheng, Yuhan, Anqi Wang, Jiaping Wu, and Jiang Chen. 2017. "Object- and pixel-based classifications of macroalgae farming area with high spatial resolution imagery. " Geocarto Int. 2017:1-25.

Zhu, Zhe, and Curtis E. Woodcock. 2014. "Continuous change detection and classification of land cover using all available Landsat data." Remote Sens. Environ. 144:152-71. 
Figure captions

Figure 1. Study area: (a) Location of Cangnan County, Zhejiang, China; (b) A ground view of Porphyra farms in Dayu Bay; (c) Detailed view of Porphyra farms.

Figure 2. Traditional classification trees of Porphyra farm identification resulting from two images: (a) Image I obtained on January 30, 2014; (b) Image II obtained on January 21, 2015. The first value in parentheses represents the number of correctly identified objects, while the second value represents the number of misclassified objects. ROI, region of interest; PC3, principal component 3.

Figure 3. Correlations between ranked features of selected objects for Image I acquired on January 30 , 2014 and Image II acquired on January 21, 2015. ML2, Mean Layer 2; PC3, principal component 3. ML2 has expanded reflectance scale and PC3 is a unitless variable.

Figure 4. Results of traditional classification trees (CT)s and modified CT: (a) traditional CT for Image I acquired on January 30, 2014; (b) Traditional CT for Image II acquired on January 21, 2015; (c)

Modified

CT for Image II.

Figure 5. Classification results of Image III acquired on November 5, 2014: (a) traditional classification tree (CT); (b) Modified CT. 

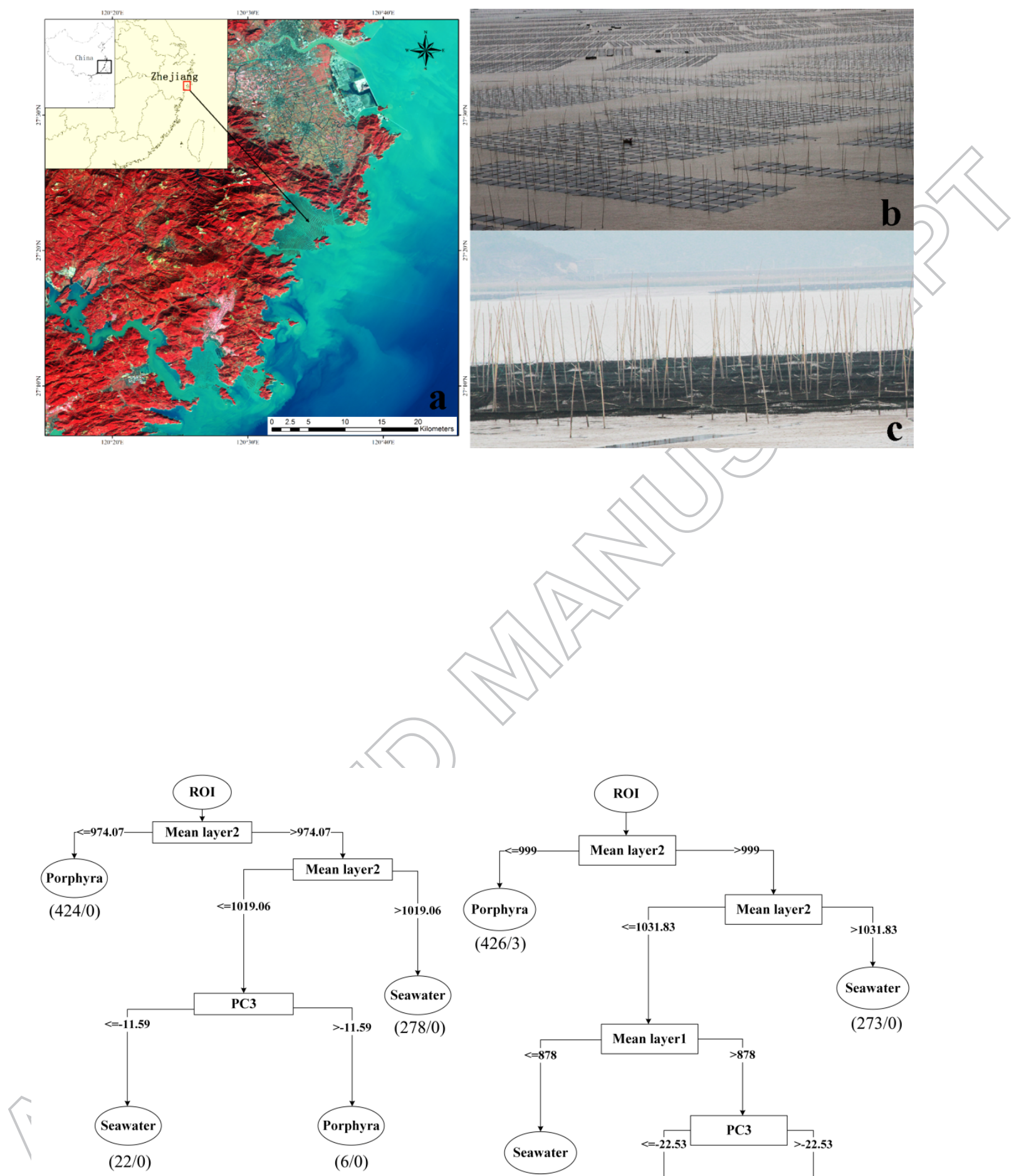

a
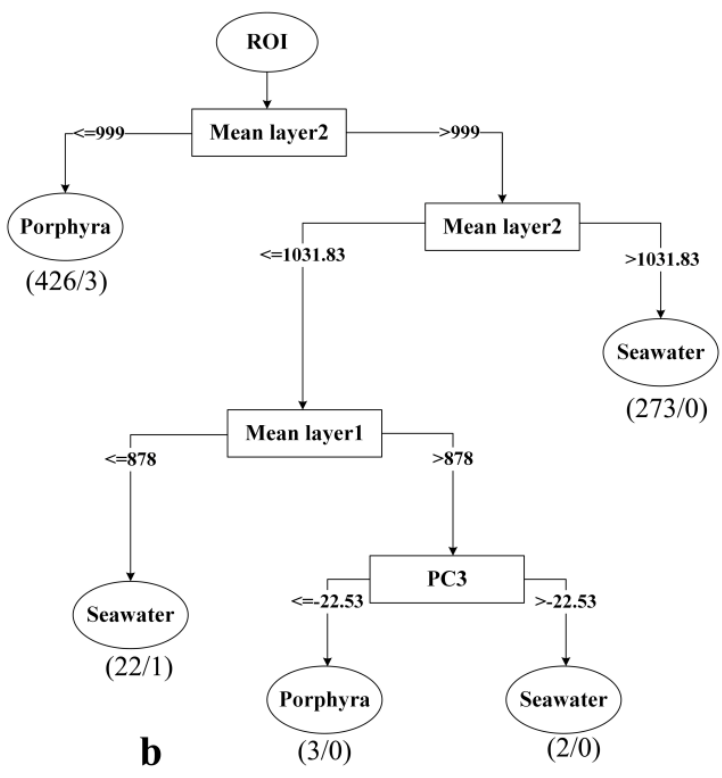


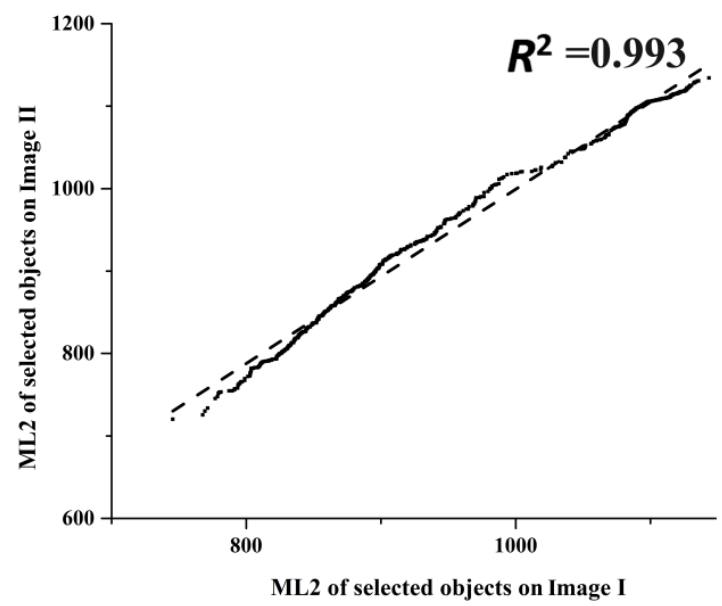

$\mathbf{a}$

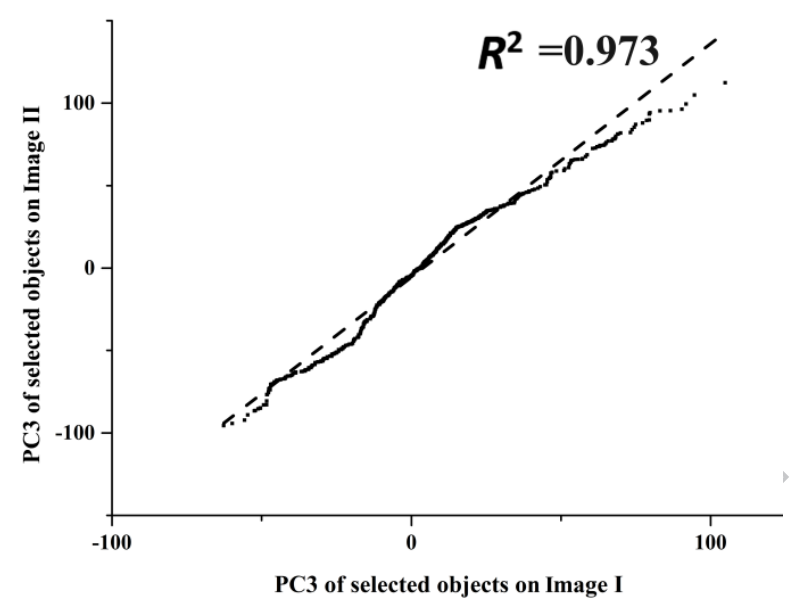

b

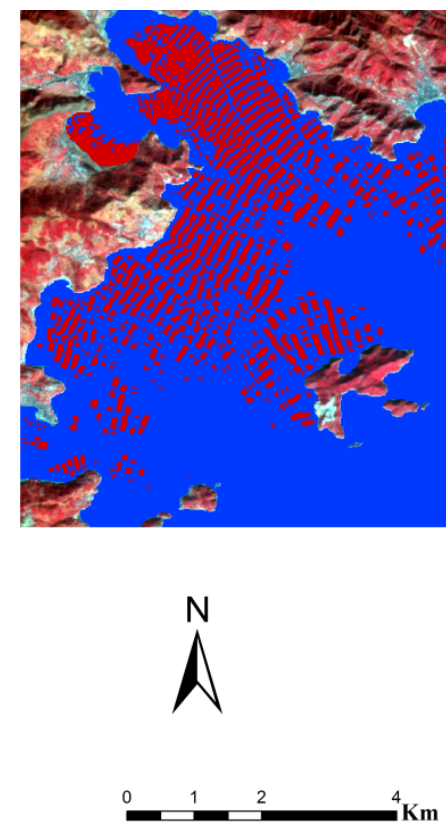

Porphyra farms

Seawater
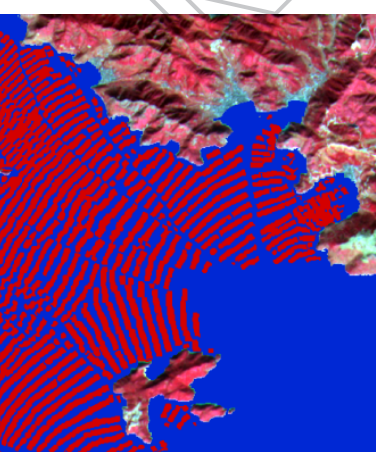

$\leq$

1)
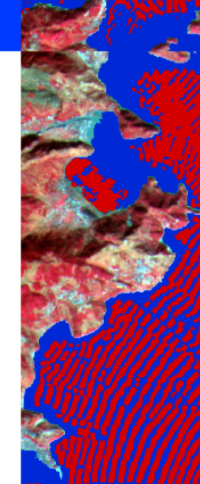


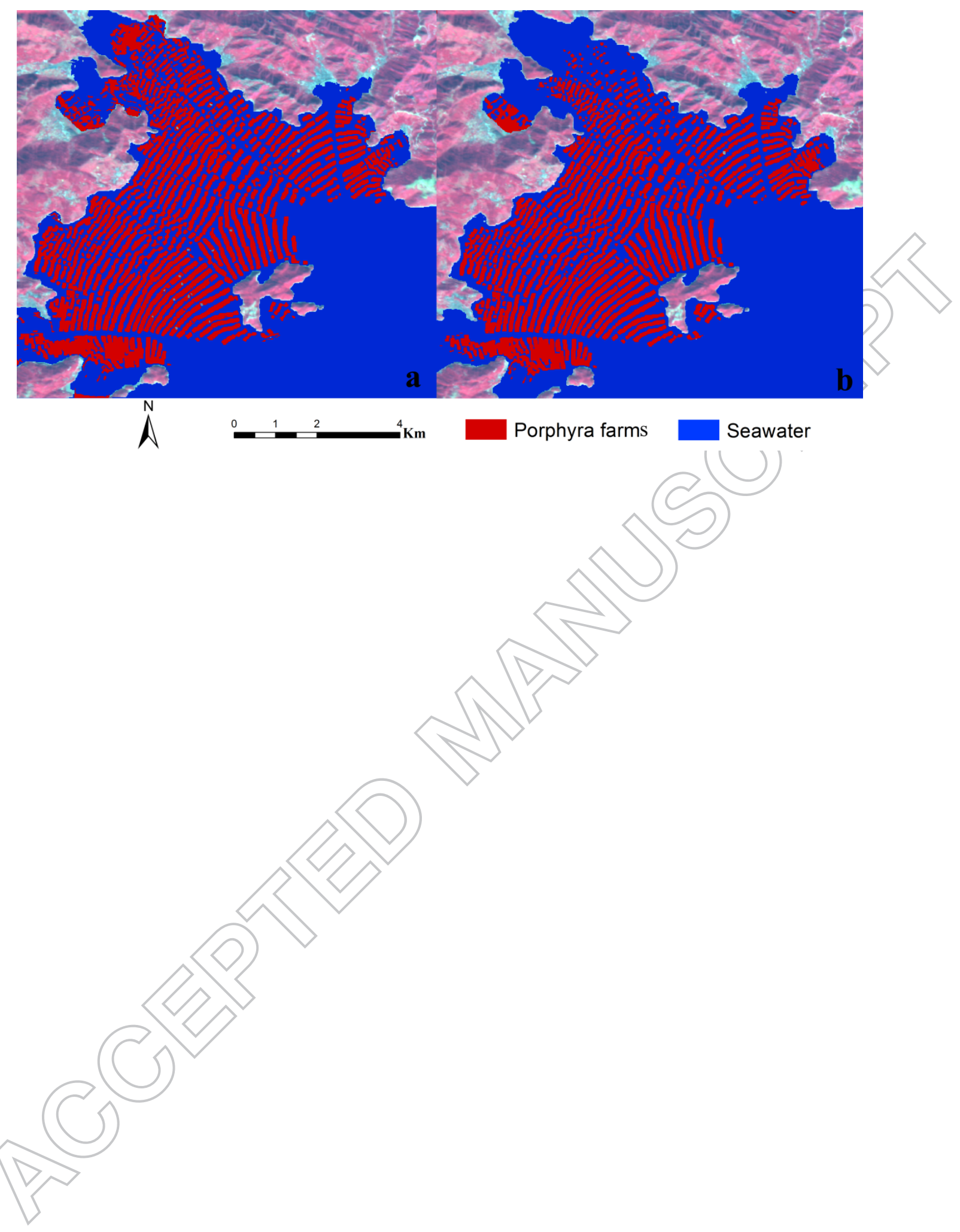


Table 1. Date, spatial resolution, and band width of the GF-1 images used in this study

\begin{tabular}{|c|c|c|c|c|}
\hline & & Image I & Image II & Image III \\
\hline \multicolumn{2}{|l|}{ Date } & $2014-1-30$ & $2015-1-21$ & $2014-11-5$ \\
\hline \multicolumn{2}{|c|}{ Spatial resolution (m) } & 16 & 16 & 16 \\
\hline \multirow[t]{4}{*}{ Band width (nm) } & B1 & & $450-520$ & \\
\hline & B2 & & $520-590$ & \\
\hline & B3 & & $630-690$ & \\
\hline & B4 & & $770-890$ & \\
\hline
\end{tabular}

Table 2. Equations and modified thresholds of features for Image I (January 30, 2014) and Image II (January 21, 2015).

\begin{tabular}{lllll}
\hline Spectral Feature & Equation & $\begin{array}{l}\text { Original } \\
\text { threshold }\end{array}$ & $\begin{array}{l}\text { Modified } \\
\text { threshold } \\
\text { (Image } \\
\text { (Image }\end{array}$ \\
\hline Mean layer 2 $(\mathrm{ML})_{2}$ & $(\mathrm{ML})_{2 H}=1.0571 \times(\mathrm{ML})_{2 I^{-}}$ & 0.993 & 974.07 & $\begin{array}{l}971.87 \\
\text { II) }\end{array}$ \\
& 57.824 \\
Principal component 3 & $(\mathrm{PC})_{3 H}=1.4153 \times(\mathrm{PC})_{3 I^{-}}$ & 0.973 & -11.59 & -21.83 \\
$(\mathrm{PC})_{3}$ & 5.4252 & & & \\
\hline
\end{tabular}

Note that the subscript $I$ represents Image I and the subscript II represents Image II. 
Table 3. Confusion matrix for three classification trees (CT)s.

\begin{tabular}{llllll}
\hline CT & Porphyra & $\begin{array}{l}\text { Sea- } \\
\text { farms }\end{array}$ & $\begin{array}{l}\text { Overall } \\
\text { waccuracy } \\
(\%)\end{array}$ & $\begin{array}{l}\text { Kappa } \\
\text { coefficient }\end{array}$ \\
\hline Traditional & Porphyra farms & 769 & 72 & & \\
CT for & Seawater & 41 & 528 & 92.0 & \\
image I & Producer accuracy (\%) & 94.9 & 88.0 & & \\
& User accuracy (\%) & 91.4 & 92.8 & & \\
\hline Traditional & Porphyra farms & 804 & 76 & & \\
CT for & Seawater & 6 & 524 & \\
image II & Producer accuracy (\%) & 99.3 & 87.3 & \\
& User accuracy (\%) & 91.4 & 99.1 & \\
\hline Modified & Porphyra farms & 799 & 87 & & \\
CT for & Seawater & 11 & 513 & 93.1 & \\
image II & Producer accuracy (\%) & 98.6 & 85.5 & & \\
& User accuracy (\%) & 90.2 & 97.9 & & \\
\hline
\end{tabular}

